Supporting information

\title{
3-Substituted 7-Phenyl-Pyrroloquinolinones Show Potent Cytotoxic Activity in Human Cancer Cell Lines
}

\author{
Venusia Gasparotto*§ ${ }^{\S}$ Ignazio Castagliuolo ${ }^{\S}$, and Maria Grazia Ferlin** \\ ${ }^{\S}$ Department of Histology, Microbiology and Medical Biotechnologies and * Department of \\ Pharmaceutical Sciences, University of Padova, Italy
}

Contents:

- yield, mp, rf and spectral data for compounds 1-32

- cell lines and culture conditions

- FACS hystograms

- table of elemental analyses

1-Methyl-5-nitro-1H-indole (1). Yield 99\%; yellow solid; mp $161{ }^{\circ} \mathrm{C}$ (methanol); rf 0.52 (eluent toluene/n-hexane 1:1); ${ }^{1} \mathrm{H}$ NMR $\left(\right.$ DMSO-d $\left._{6}\right) \delta 3.90\left(\mathrm{~s}, 3 \mathrm{H}, \mathrm{CH}_{3}\right), \mathbf{6 . 7 7}\left(\mathrm{d}, 1 \mathrm{H}, \mathrm{J}_{3,2}=3.1 \mathrm{~Hz}, 3-\mathrm{H}\right)$, $\mathbf{7 . 6 2}\left(\mathrm{d}, 1 \mathrm{H}, \mathrm{J}_{2,3}=3.1 \mathrm{~Hz}, 2-\mathrm{H}\right), \mathbf{7 . 6 7}\left(\mathrm{d}, 1 \mathrm{H}, \mathrm{J}_{7,6}=9.0 \mathrm{~Hz}, 7-\mathrm{H}\right), \mathbf{8 . 0 6}\left(\mathrm{dd}, 1 \mathrm{H}, \mathrm{J}_{6,7}=9.0 \mathrm{~Hz}, \mathrm{~J}_{6,4}=2.1\right.$ $\mathrm{Hz}, 6-\mathrm{H}), \mathbf{8 . 6 0}\left(\mathrm{d}, 1 \mathrm{H}, \mathrm{J}_{4,6}=2.1 \mathrm{~Hz}, 4-\mathrm{H}\right)$.

1-Ethyl-5-nitro-1H-indole (2). Yield 99\%; yellow solid; mp $94^{\circ} \mathrm{C}$ (methanol); rf 0.51 (eluent toluene/n-hexane 1:1); ${ }^{1} \mathrm{H}$ NMR $\left(\mathrm{DMSO}_{-} \mathrm{d}_{6}\right) \delta \mathbf{0 . 5 5}\left(\mathrm{t}, 3 \mathrm{H}, \mathrm{J}=7.1 \mathrm{~Hz}, \mathrm{CH}_{3}\right), 3.47$ (q, 2H, J = 7.1 Hz. $\left.\mathrm{CH}_{2}\right), \mathbf{5 . 9 3}\left(\mathrm{d}, 1 \mathrm{H}, \mathrm{J}_{3,2}=3.3 \mathrm{~Hz}, 2-\mathrm{H}\right), \mathbf{6 . 8 7}(\mathrm{m}, 2 \mathrm{H}, 7-\mathrm{H}$ and $3-\mathrm{H}), \mathbf{7 . 2 0}\left(\mathrm{dd}, 1 \mathrm{H}, \mathrm{J}_{6,7}=9.0 \mathrm{~Hz}\right.$, $\left.\mathrm{J}_{6,4}=2.1 \mathrm{~Hz}, 6-\mathrm{H}\right), \mathbf{7 . 7 4}\left(\mathrm{d}, 1 \mathrm{H}, \mathrm{J}_{4,6}=2.1 \mathrm{~Hz}, 4-\mathrm{H}\right)$.

1-Propyl-5-nitro-1H-indole (3). Yield 92\%; yellow thick liquid; rf 0.57 (eluente toluene/n-hexane /ethyl acetate 1:1:0.3); ${ }^{1} \mathrm{H}$ NMR $\left(\mathrm{DMSO}_{-} \mathrm{d}_{6}\right) \delta \mathbf{0 . 8 3}\left(\mathrm{t}, 3 \mathrm{H}, \mathrm{J}=7.34 \mathrm{~Hz}, \mathrm{CH}_{3}\right), \mathbf{1 . 7 9}(\mathrm{m}, 2 \mathrm{H}, \mathrm{J}=7.25$ $\left.\mathrm{Hz}, \mathrm{CH}_{2}\right), 4.23\left(\mathrm{t}, 2 \mathrm{H}, \mathrm{J}=7.20 \mathrm{~Hz}, \mathrm{CH}_{2}\right), \mathbf{6 . 7 5}\left(\mathrm{d}, 1 \mathrm{H}, \mathrm{J}_{3,2}=3.05 \mathrm{~Hz}, 3-\mathrm{H}\right), \mathbf{7 . 6 5}\left(\mathrm{d}, 1 \mathrm{H}, \mathrm{J}_{2 ., 3}=3.05\right.$ $\mathrm{Hz}, 2-\mathrm{H}), \mathbf{7 . 7 0}\left(\mathrm{d}, 1 \mathrm{H}, \mathrm{J}_{7,6}=8.96 \mathrm{~Hz}, 7-\mathrm{H}\right), \mathbf{8 . 0 1}\left(\mathrm{dd}, 1 \mathrm{H}, \mathrm{J}_{6,7}=8.96 \mathrm{~Hz}, \mathrm{~J}_{6,4}=2.29 \mathrm{~Hz}, 6-\mathrm{H}\right) .8 .57$ $\left(\mathrm{d}, 1 \mathrm{H}, \mathrm{J}_{4,6}=2.29 \mathrm{~Hz}, 4-\mathrm{H}\right)$.

\footnotetext{
* To whom correspondence should be addressed. Prof. Maria Grazia Ferlin, Department of Pharmaceutical Sciences, School of Pharmacy, University of Padova, Via Marzolo 5, 35131 Padova, Italy; Tel: 00390498271603; Fax: 00390498275366: e-mail: mariagrazia.ferlin@unipd.it
} 
1-Butyl-5-nitro-1H-indole (4).Yield 95\%; yellow thick liquid; rf 0.61(eluent toluene/n-hexane /ethyl acetate 1:1:0.3); ${ }^{1} \mathrm{H}$ NMR $\left(\mathrm{DMSO}_{6}\right) \delta \mathbf{0 . 8 6}\left(\mathrm{t}, 3 \mathrm{H}, \mathrm{J}=7.34 \mathrm{~Hz}, \mathrm{CH}_{3}\right), \mathbf{1 . 2 2}\left(\mathrm{m}, 2 \mathrm{H}, \mathrm{CH}_{2}\right)$, $1.74\left(\mathrm{~m}, 2 \mathrm{H}, \mathrm{CH}_{2}\right), \mathbf{4 . 2 5}\left(\mathrm{t}, 2 \mathrm{H}, \mathrm{J}=7.06 \mathrm{~Hz}, \mathrm{CH}_{2}\right), \mathbf{6 . 7 4}\left(\mathrm{d}, 1 \mathrm{H}, \mathrm{J}_{3,2}=3.24 \mathrm{~Hz}, 3-\mathrm{H}\right), \mathbf{7 . 6 4}\left(\mathrm{d}, 1 \mathrm{H}, \mathrm{J}_{2,3}\right.$ $=3.24 \mathrm{~Hz}, 2-\mathrm{H}), \mathbf{7 . 6 8}\left(\mathrm{d}, 1 \mathrm{H}, \mathrm{J}_{7,6}=9.16 \mathrm{~Hz}, 7-\mathrm{H}\right), \mathbf{8 . 0 1}\left(\mathrm{dd}, 1 \mathrm{H}, \mathrm{J}_{6,7}=9.16 \mathrm{~Hz}, \mathrm{~J}_{6,4}=2.00 \mathrm{~Hz}, 6-\mathrm{H}\right)$, $8.55\left(\mathrm{~d}, 1 \mathrm{H}, \mathrm{J}_{4,6}=2.10 \mathrm{~Hz}, 4-\mathrm{H}\right)$.

1-Pentyl-5-nitro-1H-indole (5). Yield 85\%; yellow-orange solid; mp 53-55 ${ }^{\circ}$; rf 0.75 (eluent toluene/n-hexane/ethyl acetate 1:1:0.3); ${ }^{1} \mathrm{H}$ NMR $\left(\right.$ DMSO-d $\left._{6}\right) \delta \mathbf{0 . 8 2}\left(\mathrm{t}, 3 \mathrm{H}, \mathrm{J}=7.16 \mathrm{~Hz}, \mathrm{CH}_{3}\right), \mathbf{1 . 2 4}$ (m, 4H, $\left.2 \mathrm{CH}_{2}\right), 1.77\left(\mathrm{~m}, 2 \mathrm{H}, \mathrm{CH}_{2}\right), 4.25\left(\mathrm{t}, 2 \mathrm{H}, \mathrm{J}=7.16 \mathrm{~Hz}, \mathrm{CH}_{2}\right), \mathbf{6 . 7 5}\left(\mathrm{dd}, 1 \mathrm{H}, \mathrm{J}_{3,2}=3,24 \mathrm{~Hz}, \mathrm{~J}_{3,4}\right.$ $=0,57 \mathrm{~Hz}, 3-\mathrm{H}), \mathbf{7 . 6 5}\left(\mathrm{d}, 1 \mathrm{H}, \mathrm{J}_{2,3}=3,24 \mathrm{~Hz}, 2-\mathrm{H}\right), \mathbf{7 . 6 9}\left(\mathrm{d}, 1 \mathrm{H}, \mathrm{J}_{7,6}=9.15 \mathrm{~Hz}, 7-\mathrm{H}\right), \mathbf{8 . 0 2}(\mathrm{dd}, 1 \mathrm{H}$, $\left.\mathrm{J}_{6,7}=8.96 \mathrm{~Hz}, \mathrm{~J}_{6,4}=2.29 \mathrm{~Hz}, 6-\mathrm{H}\right), \mathbf{8 . 5 6}\left(\mathrm{d}, 1 \mathrm{H}, \mathrm{J}_{4,6}=2.29 \mathrm{~Hz}, 4-\mathrm{H}\right)$.

1-Cyclopropylmethyl-5-nitro- $\mathbf{H}$-indole (6). Yield 99\%; brown waxy solid; mp 66-69 ${ }^{\circ} \mathrm{C}$; $\mathrm{rf} 0.95$ (eluent toluene/n-hexane/ethyl acetate 1:1:0.7); ${ }^{1} \mathrm{H}$ NMR $\left(\right.$ DMSO-d $\left._{6}\right) \delta \mathbf{0 . 4 2}\left(\mathrm{m}, 2 \mathrm{H}, \mathrm{CH}_{2}\right), \mathbf{0 . 5 2}(\mathrm{m}$, 2H, $\left.\mathrm{CH}_{2}\right), \mathbf{1 . 2 6}(\mathrm{m}, 1 \mathrm{H}, \mathrm{CH}), \mathbf{4 . 4 1}\left(\mathrm{d}, 2 \mathrm{H}, \mathrm{J}=7.01 \mathrm{~Hz}, \mathrm{CH}_{2}\right), \mathbf{6 . 7 6}\left(\mathrm{d}, 1 \mathrm{H}, \mathrm{J}_{3,2}=3.24 \mathrm{~Hz}, 3-\mathrm{H}\right), \mathbf{7 . 7 3}$ (m, 2H, 2-H and 7-H), 8.01 (dd, $\left.1 \mathrm{H}, \mathrm{J}_{6,7}=8.96 \mathrm{~Hz}, \mathrm{~J}_{6,4}=2.29 \mathrm{~Hz}, 6-\mathrm{H}\right), \mathbf{8 . 5 7}\left(\mathrm{d}, 1 \mathrm{H}, \mathrm{J}_{4,6}=2.29 \mathrm{~Hz}\right.$, 4-H).

1-Benzyl-5-nitro-1H-indole(7). Yield 96\%; yellow solid; mp 85-90 C (methanol); rf 0.66 (eluent toluene/n-hexane/ethyl acetate 1:1:0.3); ${ }^{1} \mathrm{H}$ NMR (DMSO-d $) \delta 5.53\left(s, 2 \mathrm{H}, \mathrm{CH}_{2}\right), \mathbf{7 . 2 0}(\mathrm{m}, 2 \mathrm{H}$, 2'H-6'H), 7.24 (m, 3H, 3'-H, 4'-H and 5'-H), $7.68\left(\mathrm{~d}, 1 \mathrm{H}, \mathrm{J}_{3,2}=3.05 \mathrm{~Hz}, 3-\mathrm{H}\right), \mathbf{7 . 7 7}\left(\mathrm{d}, 1 \mathrm{H}, \mathrm{J}_{7,6}=\right.$ $9.06 \mathrm{~Hz}, 7-\mathrm{H}), \mathbf{8 . 0 0}\left(\mathrm{dd}, 1 \mathrm{H}, \mathrm{J}_{6,7}=8.96 \mathrm{~Hz}, \mathrm{~J}_{6,4}=2.29 \mathrm{~Hz}, \mathrm{H}-6\right), \mathbf{8 . 6 0}\left(\mathrm{d}, 1 \mathrm{H}, \mathrm{J}_{4,6}=2.29 \mathrm{~Hz}, \mathrm{H}-4\right)$.

1-Methoxymethyl-5-nitro-1H-indole (8). Yield 93\%; yellow crystalline solid; mp $80-84^{\circ} \mathrm{C}$; rf $^{0.41}$ (eluent toluene/n-hexane/ethyl acetate 1:1:0.7); ${ }^{1} \mathrm{H} \mathrm{NMR}\left(\mathrm{CDCl}_{3}\right) \delta \mathbf{3 . 2 7}\left(\mathrm{s}, 3 \mathrm{H}, \mathrm{OCH}_{3}\right), \mathbf{5 . 4 9}(\mathrm{s}$, $\left.2 \mathrm{H}, \mathrm{CH}_{2}\right), \mathbf{6 . 7 2}\left(\mathrm{d}, 1 \mathrm{H}, \mathrm{J}_{3,2}=3.24 \mathrm{~Hz}, 3-\mathrm{H}\right), \mathbf{7 . 3 3}\left(\mathrm{d}, 1 \mathrm{H}, \mathrm{J}_{2,3}=3.24 \mathrm{~Hz}, 2-\mathrm{H}\right), \mathbf{7 . 5 2}\left(\mathrm{d}, 1 \mathrm{H}, \mathrm{J}_{7,6}=9.14\right.$ $\mathrm{Hz}, 7-\mathrm{H}), \mathbf{8 . 1 5}\left(\mathrm{dd}, 1 \mathrm{H}, \mathrm{J}_{6,7}=9.25 \mathrm{~Hz}\right.$ and $\left.\mathrm{J}_{6,4}=1.90 \mathrm{~Hz}, 6-\mathrm{H}\right), \mathbf{8 . 6 0}\left(\mathrm{d}, 1 \mathrm{H}, \mathrm{J}_{4,6}=2.10 \mathrm{~Hz}, 4-\mathrm{H}\right)$. 1-Methyl-5-amino-1H-indole (9). Yield 96\%; brown solid; rf 0.44 (eluent ethyl acetate/n-hexane S2 
1:3); mp $87^{\circ} \mathrm{C} ;{ }^{1} \mathrm{H}$ NMR $\left(\mathrm{DMSO}_{-} \mathrm{d}_{6}\right) \delta 3.66\left(\mathrm{~s}, 3 \mathrm{H}, \mathrm{CH}_{3}\right), 4.46\left(\mathrm{bs}, 2 \mathrm{H}, \mathrm{NH}_{2}\right), \mathbf{6 . 1 0}\left(\mathrm{d}, 1 \mathrm{H}, \mathrm{J}_{3,2}=\right.$ 2.1Hz, 3-H), $\mathbf{6 . 5 4}\left(\mathrm{dd}, 1 \mathrm{H}, \mathrm{J}_{6,4}=1.9 \mathrm{~Hz}, \mathrm{~J}_{6,7}=8.6 \mathrm{~Hz}, 6-\mathrm{H}\right), \mathbf{6 . 6 0}\left(\mathrm{d}, 1 \mathrm{H}, \mathrm{J}_{4,6}=1.9 \mathrm{~Hz}, 4-\mathrm{H}\right), \mathbf{7 . 1 0}$ (m, 2H, 7-H and 2-H).

1-Ethyl-5-amino-1H-indole (10).Yield: 90\% ; brown tar liquid; rf 0.22 (eluent ethyl acetate/nhexane 1:3); ${ }^{1} \mathrm{H}$ NMR $\left(\mathrm{DMSO}_{-} \mathrm{d}_{6}\right) \delta \mathbf{1 . 5 5}\left(\mathrm{t}, 3 \mathrm{H}, \mathrm{J}=7.3 \mathrm{~Hz}, \mathrm{CH}_{3}\right), \mathbf{4 . 3 0}\left(\mathrm{q}, 2 \mathrm{H}, \mathrm{J}=7.3 \mathrm{~Hz}, \mathrm{CH}_{2}\right.$ ), 4.70 (bs, $\left.2 \mathrm{H}, \mathrm{NH}_{2}\right), \mathbf{6 . 3 8}\left(\mathrm{dd}, 1 \mathrm{H}, \mathrm{J}_{3,2}=3.1 \mathrm{~Hz}\right.$ and $\left.\mathrm{J}_{3,7}=0.7 \mathrm{~Hz}, 3-\mathrm{H}\right), \mathbf{6 . 8 0}\left(\mathrm{dd}, 1 \mathrm{H}, \mathrm{J}_{6,7}=8.6 \mathrm{~Hz}\right.$, $\left.\mathrm{J}_{6,4}=2.3 \mathrm{~Hz}, 6-\mathrm{H}\right), \mathbf{6 . 9 6}\left(\mathrm{d}, 1 \mathrm{H}, \mathrm{J}_{4,6}=2.2 \mathrm{~Hz}, 4-\mathrm{H}\right), \mathbf{3 . 3 8}(\mathrm{m}, 2 \mathrm{H}, 2-\mathrm{H}$ and $7-\mathrm{H})$.

1-Propyl-5-amino-1H-indole (11). Yield 92\%; brown thik liquid; rf 0,59 (eluent dichloromethane/ethyl acetate 8:2); ${ }^{1} \mathrm{H}$ NMR $\left(\mathrm{DMSO}_{-} \mathrm{d}_{6}\right) \delta \mathbf{0 . 8 3}\left(\mathrm{t}, 3 \mathrm{H}, \mathrm{J}=7.16 \mathrm{~Hz}, \mathrm{CH}_{3}\right), \mathbf{1 . 7 9}(\mathrm{m}$, $\left.2 \mathrm{H}, \mathrm{CH}_{2}\right), \mathbf{3 . 9 8}\left(\mathrm{t}, 2 \mathrm{H}, \mathrm{J}=6.96 \mathrm{~Hz}, \mathrm{CH}_{2}\right), \mathbf{4 . 4 2}\left(\mathrm{bs}, 2 \mathrm{H}, \mathrm{NH}_{2}\right), \mathbf{6 . 1 0}\left(\mathrm{dd}, 1 \mathrm{H}, \mathrm{J}_{3,2}=3.05 \mathrm{~Hz}, \mathrm{~J}_{3,7}=\right.$ $0.76 \mathrm{~Hz}, 3-\mathrm{H}), \mathbf{6 . 5 2}\left(\mathrm{dd}, 1 \mathrm{H}, \mathrm{J}_{6,7}=8.58 \mathrm{~Hz}\right.$ and $\left.\mathrm{J}_{6,4}=2.10 \mathrm{~Hz}, 6-\mathrm{H}\right), \mathbf{6 . 6 8}\left(\mathrm{dd}, 1 \mathrm{H}, \mathrm{J}_{4,6}=2.10 \mathrm{~Hz}\right.$ and $\left.\mathrm{J}_{4,7}=0,57 \mathrm{~Hz}, 4-\mathrm{H}\right), \mathbf{7 . 1 3}(\mathrm{m}, 2 \mathrm{H}, 2-\mathrm{H}$ and $7-\mathrm{H})$.

1-Butyl-5-amino-1H-indole (12). Yield 91\%; brown thik liquid; rf 0,43 (eluent dichoromethane/ethyl acetate 8:2); ${ }^{1} \mathrm{H}$ NMR $\left(\mathrm{DMSO}_{-} \mathrm{d}_{6}\right) \delta \mathbf{0 . 8 7}\left(\mathrm{t}, 3 \mathrm{H}, \mathbf{J}=7.34 \mathrm{~Hz}, \mathrm{CH}_{3}\right), \mathbf{1 . 2 2}(\mathrm{m}$, 2H, $\left.\mathrm{CH}_{2}\right), \mathbf{1 . 6 7}\left(\mathrm{m}, 2 \mathrm{H}, \mathrm{CH}_{2}\right), 4.02\left(\mathrm{t}, 2 \mathrm{H}, \mathrm{J}=6.96 \mathrm{~Hz}, \mathrm{CH}_{2}\right), \mathbf{4 . 5 0}\left(\mathrm{bs}, 2 \mathrm{H}, \mathrm{NH}_{2}\right), \mathbf{6 . 0 9}\left(\mathrm{dd}, 1 \mathrm{H}, \mathrm{J}_{3,2}\right.$ $=3.08 \mathrm{~Hz}$ and $\left.\mathrm{J}_{3,7}=0.82 \mathrm{~Hz}, 3-\mathrm{H}\right), \mathbf{6 . 5 1}\left(\mathrm{dd}, 1 \mathrm{H}, \mathrm{J}_{6,7}=8.77 \mathrm{~Hz}\right.$ and $\left.\mathrm{J}_{6,4}=2.10 \mathrm{~Hz}, 6-\mathrm{H}\right), \mathbf{6 . 6 7}(\mathrm{dd}$, $1 \mathrm{H}, \mathrm{J}_{4,6}=2.10 \mathrm{~Hz}$ and $\left.\mathrm{J}_{4,7}=0.57 \mathrm{~Hz}, 4-\mathrm{H}\right), 7.12(\mathrm{~m}, 2 \mathrm{H}, 2-\mathrm{H}$ and $7-\mathrm{H})$.

1-Pentyl-5-amino-1H-indole (13). Yield 89\%; dark thik liquid; rf 0,58 (eluent dichloromethane/ethyl acetate 8:2; ${ }^{1} \mathrm{H}$ NMR $\left(\mathrm{DMSO}^{\left.-\mathrm{d}_{6}\right)} \delta \mathbf{0 . 8 3}\left(\mathrm{t}, 3 \mathrm{H}, \mathbf{J}=7.06 \mathrm{~Hz}, \mathrm{CH}_{3}\right), \mathbf{1 . 2 4}(\mathrm{m}\right.$, 4H, $\left.2 \mathrm{CH}_{2}\right), \mathbf{1 . 6 9}\left(\mathrm{m}, 2 \mathrm{H}, \mathrm{CH}_{2}\right), 4.01\left(\mathrm{t}, 2 \mathrm{H}, \mathrm{J}=7.06 \mathrm{~Hz}, \mathrm{CH}_{2}\right), 4.48\left(\mathrm{bs}, 2 \mathrm{H}, \mathrm{NH}_{2}\right), \mathbf{6 . 0 9}(\mathrm{dd}, 1 \mathrm{H}$, $\left.\mathrm{J}_{3,2}=3,05 \mathrm{~Hz}, \mathrm{~J}_{3,7}=0.76 \mathrm{~Hz}, 3-\mathrm{H}\right), \mathbf{6 . 5 1}\left(\mathrm{dd}, 1 \mathrm{H}, \mathrm{J}_{6,7}=8.58 \mathrm{~Hz}\right.$ and $\left.\mathrm{J}_{6,4}=2.10 \mathrm{~Hz}, 6-\mathrm{H}\right), \mathbf{6 . 6 7}(\mathrm{dd}$, $1 \mathrm{H}, \mathrm{J}_{4,6}=1.82 \mathrm{~Hz}$ and $\left.\mathrm{J}_{4,7}=0.57 \mathrm{~Hz}, 4-\mathrm{H}\right), \mathbf{7 . 1 1}(\mathrm{m}, 2 \mathrm{H}, \mathrm{H}-2$ and $\mathrm{H}-7)$.

1-Cyclopropylmethyl-5-amino-1H-indole (14). Yield 62\%; brown thik liquid; rf 0.46 (eluent chloroform/ethyl acetate 8:2); ${ }^{1} \mathrm{H}$ NMR $\left(\mathrm{DMSO}_{\mathrm{d}}\right) \delta \mathbf{0 . 3 2}\left(\mathrm{m}, 2 \mathrm{H}, \mathrm{CH}_{2}\right), \mathbf{0 . 4 8}\left(\mathrm{m}, 2 \mathrm{H}, \mathrm{CH}_{2}\right), \mathbf{1 . 1 8}$ 
(m, $1 \mathrm{H}, \mathrm{CH}), \mathbf{3 . 9 0}\left(\mathrm{d}, 2 \mathrm{H}, \mathrm{J}=7.06 \mathrm{~Hz}, \mathrm{CH}_{2}\right), \mathbf{4 . 4 9}\left(\mathrm{bs}, 2 \mathrm{H}, \mathrm{NH}_{2}\right), \mathbf{6 . 1 1}\left(\mathrm{dd}, 1 \mathrm{H}, \mathrm{J}_{3,2}=3.05 \mathrm{~Hz}\right.$ and $\left.\mathbf{J}_{3,7}=0.76 \mathrm{~Hz}, 3-\mathrm{H}\right), \mathbf{6 . 5 2}\left(\mathrm{dd}, 1 \mathrm{H}, \mathrm{J}_{6,7}=8.58 \mathrm{~Hz}\right.$ and $\left.\mathrm{J}_{6,4}=2.09 \mathrm{~Hz}, 6-\mathrm{H}\right), \mathbf{6 . 6 7}\left(\mathrm{dd}, 1 \mathrm{H}, \mathrm{J}_{4,6}=2.10\right.$ $\left.\mathrm{Hz}, \mathrm{J}_{4,3}=0.57 \mathrm{~Hz}, 4-\mathrm{H}\right), \mathbf{7 . 1 6}\left(\mathrm{d}, 1 \mathrm{H}, \mathrm{J}_{7,6}=8.58 \mathrm{~Hz}, 7-\mathrm{H}\right), 7.19\left(\mathrm{~d}, 1 \mathrm{H}, \mathrm{J}_{2,3}=2.98 \mathrm{~Hz}, 2-\mathrm{H}\right)$.

1-Benzyl-5-amino-1H-indole (15). Yield 76\%; brown waxy liquid; rf 0.51 (eluent dichloromethane/ethyl acetate 8:2); ${ }^{1} \mathrm{H}$ NMR $\left(\mathrm{DMSO}_{\mathrm{d}}\right) \delta \mathbf{4 . 2 5}\left(\mathrm{bs}, 2 \mathrm{H}, \mathrm{NH}_{2}\right), \mathbf{5 . 2 0}\left(\mathrm{s}, 2 \mathrm{H}, \mathrm{CH}_{2}\right)$, $\mathbf{6 . 1 8}\left(\mathrm{d}, 1 \mathrm{H}, \mathrm{J}_{3,2}=2.86 \mathrm{~Hz}, 3-\mathrm{H}\right), \mathbf{6 . 5 0}\left(\mathrm{dd}, 1 \mathrm{H}, \mathrm{J}_{6,7}=8.58 \mathrm{~Hz}, \mathrm{~J}_{6,4}=2.09 \mathrm{~Hz}, 6-\mathrm{H}\right), \mathbf{6 . 7 0}\left(\mathrm{d}, 1 \mathrm{H}, \mathrm{J}_{4,6}\right.$ $=2.09 \mathrm{~Hz}, 4-\mathrm{H}), 7.15\left(\mathrm{~m}, 2 \mathrm{H}, 2^{\prime}-\mathrm{H}\right.$ and 6'-H), $7.25\left(\mathrm{~m}, 3 \mathrm{H}, 3^{\prime}-\mathrm{H}, 4^{\prime}-\mathrm{H}\right.$ and 5'-H).

1-Metoxymethyl-5-amino-1H-indole (16). Yied 89\%; dark thik liquid; rf 0.23 (eluent dichloromethane/ethyl acetate 8:2); ${ }^{1} \mathrm{H}$ NMR $\left(\mathrm{DMSO}_{-} \mathrm{d}_{6}\right) \delta \mathbf{3 . 1 2}\left(\mathrm{s}, 3 \mathrm{H}, \mathrm{OCH}_{3}\right), \mathbf{4 . 5 3}\left(\mathrm{bs}, 2 \mathrm{H}, \mathrm{NH}_{2}\right)$, $\mathbf{5 . 3 7}\left(\mathrm{s}, 2 \mathrm{H}, \mathrm{CH}_{2}\right), \mathbf{6 . 1 9}\left(\mathrm{dd}, 1 \mathrm{H}, \mathrm{J}_{3,2}=3.05 \mathrm{~Hz}\right.$ and $\left.\mathrm{J}_{3,7}=0.76 \mathrm{~Hz}, 3-\mathrm{H}\right), \mathbf{6 . 5 4}\left(\mathrm{dd}, 1 \mathrm{H}, \mathrm{J}_{6,7}=8.46 \mathrm{~Hz}\right.$ and $\left.\mathrm{J}_{6,4}=2.03 \mathrm{~Hz}, 6-\mathrm{H}\right), \mathbf{6 . 6 9}\left(\mathrm{dd}, 1 \mathrm{H}, \mathrm{J}_{4,6}=1.72 \mathrm{~Hz}\right.$ and $\left.\mathrm{J}_{4,7}=0,57 \mathrm{~Hz}, 4-\mathrm{H}\right), \mathbf{7 . 2 1}\left(\mathrm{d}, 1 \mathrm{H}, \mathrm{J}_{7,6}=\right.$ 8.77 Hz, 7-H), $7.24\left(\mathrm{~d}, 1 \mathrm{H}, \mathrm{J}_{2,3}=3.05 \mathrm{~Hz}, 2-\mathrm{H}\right)$.

Ethyl 3-phenyl-3-(1-methyl-1H-indol-5-ylamino)-acrylate (17). Yield 55\%; brown solid; mp 120$122^{\circ} \mathrm{C}$; rf 0.83 (eluent dichloromethane/ethyl acetate 9:1); ${ }^{1} \mathrm{H}$ NMR (DMSO- $\left.\mathrm{d}_{6}\right): \delta \mathbf{1 . 2 3}(\mathrm{t}, 3 \mathrm{H}, \mathrm{J}=$ 7.06 Hz, $\left.\mathrm{CH}_{3}\right), \mathbf{3 . 6 9}\left(\mathrm{s}, 3 \mathrm{H}, 2 \mathrm{CH}_{3}\right), 4.13\left(\mathrm{q}, 2 \mathrm{H}, \mathrm{J}=7.06 \mathrm{~Hz}, \mathrm{CH}_{2}\right), \mathbf{4 . 8 3}(\mathrm{s}, 1 \mathrm{H}, \mathrm{CH}), \mathbf{6 . 2 2}(\mathrm{d}, 1 \mathrm{H}$, $\left.\mathrm{J}_{3,2}=3.05 \mathrm{~Hz}, 2-\mathrm{H}\right), \mathbf{6 . 6 2}\left(\mathrm{dd}, 1 \mathrm{H}, \mathrm{J}_{6,7}=9.15 \mathrm{~Hz}\right.$ and $\left.\mathrm{J}_{6,4}=2.10 \mathrm{~Hz}, 6-\mathrm{H}\right), \mathbf{6 . 9 8}\left(\mathrm{d}, 1 \mathrm{H}, \mathrm{J}_{4,6}=1.33\right.$ $\mathrm{Hz}, 4-\mathrm{H}), 7.19\left(\mathrm{~d}, 1 \mathrm{H}, \mathrm{J}_{7,6}=8.77 \mathrm{~Hz}, 7-\mathrm{H}\right), 7.24\left(\mathrm{~d}, 1 \mathrm{H}, \mathrm{J}_{3,2}=3.05 \mathrm{~Hz}, 3-\mathrm{H}\right) \mathbf{7 . 3 0}(\mathrm{m}, 5 \mathrm{H}$, phenyl), $10.25(\mathrm{~s}, 1 \mathrm{H}, \mathrm{NH})$.

Ethyl 3-phenyl-3-(1-ethyl-1H-indol-5-ylamino)-acrylate (18). Yield 60\%; brown soid; mp 94$96^{\circ} \mathrm{C}$; rf 0.92 (eluent dichloromethane/ethyl acetate 9:1); ${ }^{1} \mathrm{H}$ NMR $\left(\right.$ DMSO-d $\left.\mathrm{d}_{6}\right) \delta \mathbf{1 . 2 3}(\mathrm{t}, 3 \mathrm{H}, \mathrm{J}=$ $\left.7.25 \mathrm{~Hz}, \mathrm{CH}_{3}\right), \mathbf{1 . 2 9}\left(\mathrm{t}, 3 \mathrm{H}, 2 \mathrm{CH}_{3}\right), \mathbf{4 . 1 1}\left(\mathrm{m}, 4 \mathrm{H}, \mathrm{J}=7.06 \mathrm{~Hz}, 2 \mathrm{xCH}_{2}\right), \mathbf{4 . 8 3}(\mathrm{s}, 1 \mathrm{H}, \mathrm{CH}), \mathbf{6 . 2 2}(\mathrm{d}$, $\left.1 \mathrm{H}, \mathrm{J}_{3,2}=3.05 \mathrm{~Hz}, 3-\mathrm{H}\right), \mathbf{6 . 6 2}\left(\mathrm{dd}, 1 \mathrm{H}, \mathrm{J}_{6,7}=8.65 \mathrm{~Hz}\right.$ and $\left.\mathrm{J}_{6,4}=1.97 \mathrm{~Hz}, 6-\mathrm{H}\right), \mathbf{6 . 9 7}\left(\mathrm{d}, 1 \mathrm{H}, \mathrm{J}_{4,6}=\right.$ $1.91 \mathrm{~Hz}, 4-\mathrm{H}), \mathbf{7 . 2 3}\left(\mathrm{d}, 1 \mathrm{H}, \mathrm{J}_{7,6}=8.77 \mathrm{~Hz}, 7-\mathrm{H}\right), \mathbf{7 . 3 1}$ (m, 6H, 2-H and phenyl), 10.25 (s, 1H, NH). Ethyl 3-phenyl-3-(1-propyl-1H-indol-5-ylamino)-acrylate (19). Yield 65\%; yellow thick liquid; 
rf 0.60 (eluene dichloromethane); ${ }^{1} \mathrm{H}-\mathrm{NMR}\left(\mathrm{DMSO}_{6}\right) \delta \mathbf{0 . 8 0}\left(\mathrm{t}, 3 \mathrm{H}, \mathrm{J}=7.25 \mathrm{~Hz}, \mathrm{CH}_{3}\right), \mathbf{1 . 2 3}(\mathrm{t}$, $\left.3 \mathrm{H}, \mathrm{J}=7.25 \mathrm{~Hz}, \mathrm{CH}_{3}\right), \mathbf{1 . 7 0}\left(\mathrm{m}, 2 \mathrm{H}, \mathrm{CH}_{2}\right), \mathbf{4 . 0 2}\left(\mathrm{t}, 2 \mathrm{H}, \mathrm{J}=7.06 \mathrm{~Hz}, \mathrm{CH}_{2}\right), \mathbf{4 . 1 3}(\mathrm{q}, 2 \mathrm{H}, \mathrm{J}=7.25 \mathrm{~Hz}$, $\left.\mathrm{CH}_{2}\right), \mathbf{4 . 8 3}(\mathrm{s}, 1 \mathrm{H}, \mathrm{CH}), \mathbf{6 . 2 0}\left(\mathrm{d}, 1 \mathrm{H}, \mathrm{J}_{3,2}=3.05 \mathrm{~Hz}, 3-\mathrm{H}\right), \mathbf{6 . 6 0}\left(\mathrm{dd}, 1 \mathrm{H}, \mathrm{J}_{6,7}=8.77 \mathrm{~Hz}, \mathrm{~J}_{6,4}=2.10\right.$ Hz, 6-H), 6.97 (d, 1H, J $\left.\mathrm{H}_{4,6}=2.10 \mathrm{~Hz}, 4-\mathrm{H}\right), \mathbf{7 . 3 0}$ (m, 7H, 2-H, 7-H, and phenyl), 10.25 (s, 1H, NH).

Ethyl 3-phenyl-3-(1-butyl-1H-indol-5-ylamino)-acrylate (20). Yield 98\%; brown thik liquid;; rf 0.88 (eluent dichloromethane/ethyl acetate 8:2); ${ }^{1} \mathrm{H}$ NMR $\left(\right.$ DMSO-d $\left._{6}\right) \delta \mathbf{1 . 0 1}(\mathrm{t}, 3 \mathrm{H}, \mathrm{J}=7.44 \mathrm{~Hz}$, $\left.\mathrm{CH}_{3}\right), \mathbf{1 . 3 2}\left(\mathrm{t}, 2 \mathrm{H}, \mathrm{J}=7.25 \mathrm{~Hz}, \mathrm{CH}_{2}\right), \mathbf{1 . 4 0}\left(\mathrm{t}, 3 \mathrm{H}, \mathrm{J}=7.06 \mathrm{~Hz}, \mathrm{CH}_{3}\right), \mathbf{4 . 2 6}\left(\mathrm{m}, 6 \mathrm{H}, 3 \mathrm{xCH}_{2}\right), \mathbf{5 . 0 0}(\mathrm{s}$, $1 \mathrm{H}, \mathrm{CH}), \mathbf{6 . 2 0}\left(\mathrm{d}, 1 \mathrm{H}, \mathrm{J}_{3,2}=3.05 \mathrm{~Hz}, 3-\mathrm{H}\right), \mathbf{6 . 6 0}\left(\mathrm{dd}, 1 \mathrm{H}, \mathrm{J}_{6,7}=8.77 \mathrm{~Hz}, \mathrm{~J}_{6,4}=2.10 \mathrm{~Hz}, 6-\mathrm{H}\right), \mathbf{6 . 9 7}$ (d, 1H, J J,6 $=2.10 \mathrm{~Hz}, 4-\mathrm{H}), \mathbf{7 . 3 0}(\mathrm{m}, 7 \mathrm{H}, 2-\mathrm{H}, 7-\mathrm{H}$ and phenyl), 10.25 (s, 1H, NH).

Ethyl 3-phenyl-3-(1-penthyl-1H-indol-5-ylamino)-acrylate (21). Yield 46\%; yellow amorphous solid; mp 88-90 ${ }^{\circ} \mathrm{C}$; Rf 0.61 (eluent dichloromethane); ${ }^{1} \mathrm{H}$ NMR (DMSO-d $\left.{ }_{6}\right) \delta \mathbf{0 . 8 0}$ (t, $3 \mathrm{H}, \mathrm{J}=7.06$ $\left.\mathrm{Hz}, \mathrm{CH}_{3}\right), \mathbf{1 . 2 0}\left(\mathrm{m}, 7 \mathrm{H}, 2 \mathrm{xCH} \mathrm{CH}_{2}\right.$ and $\left.\mathrm{CH}_{3}\right), \mathbf{1 . 6 7}\left(\mathrm{m}, 2 \mathrm{H}, \mathrm{CH}_{2}\right), \mathbf{4 . 0 4}\left(\mathrm{t}, 2 \mathrm{H}, \mathrm{J}=7.06 \mathrm{~Hz}, \mathrm{CH}_{2}\right), 4.13$ $\left(\mathrm{q}, 2 \mathrm{H}, \mathrm{J}=7.25 \mathrm{~Hz}, \mathrm{CH}_{2}\right), \mathbf{4 . 8 3}(\mathrm{s}, 1 \mathrm{H}, \mathrm{CH}), \mathbf{6 . 2 0}\left(\mathrm{d}, 1 \mathrm{H}, \mathrm{J}_{3,2}=3,05 \mathrm{~Hz}, 3-\mathrm{H}\right), \mathbf{6 . 6 0}\left(\mathrm{dd}, 1 \mathrm{H}, \mathrm{J}_{6,7}=\right.$ $\left.8.58 \mathrm{~Hz}, \mathrm{~J}_{6,4}=2.10 \mathrm{~Hz}, 6-\mathrm{H}\right), \mathbf{6 . 9 6}\left(\mathrm{d}, 1 \mathrm{H}, \mathrm{J}_{4,6}=1.91 \mathrm{~Hz}, 4-\mathrm{H}\right), \mathbf{7 . 2 2}\left(\mathrm{d}, 1 \mathrm{H}, \mathrm{J}_{7,6}=8.77 \mathrm{~Hz}, 7-\mathrm{H}\right)$, 7.30 (m, 6H, 2-H and phenyl), $10.25(\mathrm{~s}, 1 \mathrm{H}, \mathrm{NH})$.

Ethyl 3-phenyl-3-(1-cyclopropylmethyl-1H-indol-5-ylamino)-acrylate (22). Yield 78\%; orange thik liquid; rf 0.95 (eluent dichloromethane/ethyl acetate 9:1); ${ }^{1} \mathrm{H}$ NMR (DMSO-d 6 ) $\delta \mathbf{0 . 3 2}(\mathrm{td}, 2 \mathrm{H}$, $\left.\mathrm{J}=5.15 \mathrm{~Hz}, \mathrm{CH}_{2}\right), \mathbf{0 . 4 6}\left(\mathrm{m}, 5 \mathrm{H}, 2 \mathrm{xCH}_{2}\right.$ and $\left.\mathrm{CH}\right), \mathbf{1 . 2 3}\left(\mathrm{t}, 3 \mathrm{H}, \mathrm{J}=6.96 \mathrm{~Hz}, \mathrm{CH}_{3}\right), 3.81(\mathrm{~d}, 2 \mathrm{H}, \mathrm{J}=$ $\left.6.87 \mathrm{~Hz}, \mathrm{CH}_{2}\right), 4.12$ (q, 2H, J = 7.06 Hz, $\left.\mathrm{CH}_{2}\right), \mathbf{4 . 8 3}(\mathrm{s}, 1 \mathrm{H}, \mathrm{CH}), \mathbf{6 . 2 0}\left(\mathrm{d}, 1 \mathrm{H}, \mathrm{J}_{3,2}=3.05 \mathrm{~Hz}, 3-\mathrm{H}\right)$, $\mathbf{6 . 6 0}\left(\mathrm{dd}, 1 \mathrm{H}, \mathrm{J}_{6,7}=8.77 \mathrm{~Hz}\right.$ and $\left.\mathrm{J}_{6,4}=2.10 \mathrm{~Hz}, 6-\mathrm{H}\right), \mathbf{6 . 9 7}\left(\mathrm{d}, 1 \mathrm{H}, \mathrm{J}_{4,6}=2.10 \mathrm{~Hz}, 4-\mathrm{H}\right), 7.30(\mathrm{~m}, 7 \mathrm{H}$, 2-H, 7-H and phenyl), $\mathbf{1 0 . 2 5}$ (s, 1H, NH).

Ethyl 3-phenyl-3-(1-benzyl-1H-indol-5-ylamino)-acrylate (23). Yield 49\%;yellow solid; mp 144$146^{\circ} \mathrm{C} ;$ rf 0,95 (eluent dichloromethane/ethyl acetate 8:2); ${ }^{1} \mathrm{H}$ NMR $\left(\mathrm{DMSO}_{-} \mathrm{d}_{6}\right) \delta \mathbf{1 . 2 3}(\mathrm{t}, 3 \mathrm{H}, \mathrm{J}=$ 
$\left.7.06 \mathrm{~Hz}, \mathrm{CH}_{3}\right), 4.12\left(\mathrm{q}, 2 \mathrm{H}, \mathrm{J}=7.06 \mathrm{~Hz}, \mathrm{CH}_{2}\right), 4.83(\mathrm{~s}, 1 \mathrm{H}, \mathrm{CH}), \mathbf{5 . 3 1}\left(\mathrm{s}, 2 \mathrm{H}, \mathrm{CH}_{2}\right), \mathbf{6 . 2 0}(\mathrm{d}, 1 \mathrm{H}$, $\left.\mathrm{J}_{3,2}=3.05 \mathrm{~Hz}, 3-\mathrm{H}\right), \mathbf{6 . 6 0}\left(\mathrm{dd}, 1 \mathrm{H}, \mathrm{J}_{6,7}=8.77 \mathrm{~Hz}\right.$ and $\left.\mathrm{J}_{6,4}=2.10 \mathrm{~Hz}, 6-\mathrm{H}\right), \mathbf{6 . 9 7}\left(\mathrm{d}, 1 \mathrm{H}, \mathrm{J}_{4,6}=2.10 \mathrm{~Hz}\right.$, 4-H), 7.30 (m, 12H, 2-H, 7-H and 2xphenyl), 7.42 (d, 1H, J

Ethyl 3-phenyl-3-(1-methoxymethyl-1H-indol-5-ylamino)-acrylate (24). Yield 66\%; orange waxy solid; rf 0.93 (eluent dichloromethane/ethyl acetate 8:2); ${ }^{1} \mathrm{H}$ NMR (DMSO- $\left.\mathrm{d}_{6}\right) \delta \mathbf{1 . 2 0}(\mathrm{m}, 3 \mathrm{H}$, $\left.\mathrm{CH}_{3}\right), \mathbf{3 . 1 0}\left(\mathrm{s}, 3 \mathrm{H}, \mathrm{CH}_{3}\right), \mathbf{4 . 2 0}\left(\mathrm{m}, 3 \mathrm{H}, \mathrm{CH}\right.$ and $\left.\mathrm{CH}_{2}\right), \mathbf{5 . 4 1}\left(\mathrm{s}, 2 \mathrm{H}, \mathrm{CH}_{2}\right), \mathbf{6 . 2 0}\left(\mathrm{d}, 1 \mathrm{H}, \mathrm{J}_{3,2}=3.05 \mathrm{~Hz}\right.$, 3-H), $\mathbf{6 . 6 0}\left(\mathrm{dd}, 1 \mathrm{H}, \mathrm{J}_{6,7}=8.58 \mathrm{~Hz}\right.$ and $\left.\mathrm{J}_{6,4}=2.10 \mathrm{~Hz}, 6-\mathrm{H}\right), \mathbf{6 . 9 6}\left(\mathrm{d}, 1 \mathrm{H}, \mathrm{J}_{4,6}=1.91 \mathrm{~Hz}, \mathrm{H}-4\right), \mathbf{7 . 4 0}$ $\left(\mathrm{d}, 1 \mathrm{H}, \mathrm{J}_{2,3}=3.05 \mathrm{~Hz}, 2-\mathrm{H}\right) 7.30\left(\mathrm{~m}, 5 \mathrm{H}\right.$, phenyl), $7.96\left(\mathrm{~d}, 1 \mathrm{H}, \mathrm{J}_{7,6}=8.77 \mathrm{~Hz}, 7-\mathrm{H}\right), 10.25(\mathrm{~s}, 1 \mathrm{H}$, $\mathrm{NH})$.

3-Methyl-7-phenyl-3H-pyrrolo[3,2-f]quinolin-9-one (25). Yield 67\%; light brown solid ; mp > $330^{\circ} \mathrm{C}$; rf 0.83 (eluent ethyl acetate/methanol 9:1); ${ }^{1} \mathrm{H}$ NMR $\left(\right.$ DMSO $\left.\mathrm{d}_{6}\right) \delta 3.91\left(\mathrm{~s}, 3 \mathrm{H}, \mathrm{CH}_{3}\right), \mathbf{6 . 3 8}$ (bs, 1H, 8-H), $7.43\left(\mathrm{~d}, 1 \mathrm{H}, \mathrm{J}_{1,2}=2.86 \mathrm{~Hz}, 1-\mathrm{H}\right), 7.52\left(\mathrm{~d}, 1 \mathrm{H}, \mathrm{J}_{2,1}=2.86 \mathrm{~Hz}, 2-\mathrm{H}\right), 7.57$ (m, 4H, 5-H, 3'-H, 4'-H, 5'-H), 7.84 (m, 3H, 4-H, 2'-H, 6'-H), 11.64 (bs, 1H, NH); ${ }^{13} \mathrm{C}$ NMR (DMSO-d 6 ): $\delta$ 33.11 $\left(\mathrm{CH}_{3}\right), \mathbf{1 8 8 . 6 9}(\mathrm{C}=\mathrm{O})$; HR MS m/z 275.1137 [MH'].

3-Ethyl-7-phenyl-3H-pyrrolo[3,2-f]quinolin-9-one (26). Yield 66\%; yellow solid; mp 256-258 ${ }^{\circ}$; rf 0.70 (eluent ethyl acetate/methanol 9:1); ${ }^{1} \mathrm{H}$ NMR (DMSO-d 6 ) $\delta \mathbf{1 . 4 0}\left(\mathrm{t}, 3 \mathrm{H}, \mathrm{J}=7.25, \mathrm{CH}_{3}\right), \mathbf{4 . 3 1}$ $\left(\mathrm{q}, 2 \mathrm{H}, \mathrm{J}=7.25, \mathrm{CH}_{2}\right), \mathbf{6 . 3 8}(\mathrm{bs}, 1 \mathrm{H}, 8-\mathrm{H}), \mathbf{7 . 5 0}\left(\mathrm{d}, 1 \mathrm{H}, \mathrm{J}_{1,2}=2.67 \mathrm{~Hz}, 1-\mathrm{H}\right), \mathbf{7 . 5 6}(\mathrm{m}, 5 \mathrm{H}$, 5-H, 2-H, 3'-H, 4'-H, 5'-H), 7.85 (m, 3H, 4-H, 2'-H, 6'-H), 11.62 (bs, 1H, NH); ${ }^{13} \mathrm{C}$ NMR (DMSO- d $62 \delta$ 14.28 $\left(\mathrm{CH}_{3}\right), \mathbf{5 0 . 3 0}\left(\mathrm{CH}_{2}\right), \mathbf{1 8 4 . 3 8}(\mathrm{C}=\mathrm{O}) ; \mathrm{HR} \mathrm{MS} \mathrm{m} / \mathrm{z} 289.1284\left[\mathrm{MH}^{+}\right]$.

3-Propyl-7-phenyl-3H-pyrrolo[3,2-f]quinolin-9-one (27). Yield 85\%; yellow solid; mp 254$257^{\circ} \mathrm{C}$; rf 0.56 (eluent ethyl acetate/methanol 9:1); ${ }^{1} \mathrm{H}$ NMR (DMSO-d 6 ) $\delta \mathbf{0 . 8 2}(\mathrm{t}, 3 \mathrm{H}, \mathrm{J}=7.44 \mathrm{~Hz}$, $\left.\mathrm{CH}_{3}\right), \mathbf{1 . 8 0}\left(\mathrm{m}, 2 \mathrm{H}, \mathrm{CH}_{2}\right), \mathbf{4 . 2 4}\left(\mathrm{t}, 2 \mathrm{H}, \mathrm{J}=6.87 \mathrm{~Hz}, \mathrm{CH}_{2}\right), \mathbf{6 . 3 7}(\mathrm{d}, 1 \mathrm{H}, \mathrm{J}=1.71 \mathrm{~Hz}, 8-\mathrm{H}), \mathbf{7 . 4 8}(\mathrm{d}$, $\left.1 \mathrm{H}, \mathrm{J}_{1,2}=2.86 \mathrm{~Hz}, 1-\mathrm{H}\right), 7.53$ (m, 5H, 5-H, 2-H, 3'-H, 4'-H, 5'-H), 7.85 (m, 3H, 4-H, 2'-H, 6'-H), 
11.62 (bs, $1 \mathrm{H}, \mathrm{NH}) ;{ }^{13} \mathrm{C}$ NMR (DMSO-d 6$)$ : $\delta 13.93\left(\mathrm{CH}_{3}\right), \mathbf{2 6 . 4 6}\left(\mathrm{CH}_{2}\right), \mathbf{5 0 . 1 5}\left(\mathrm{N}-\mathrm{CH}_{2}\right), \mathbf{1 8 5 . 6 0}$ $(\mathrm{C}=\mathrm{O}) ; \mathrm{HR}$ MS m/z $303.1531[\mathrm{MH}+]$.

3-Butyl-7-phenyl-3H-pyrrolo[3,2-f]quinolin-9-one (28). Yield 64\%; light brown solid; mp 234$237^{\circ} \mathrm{C}$; rf 0.74 (eluent ethyl acetate/methanol 8:2); ${ }^{1} \mathrm{H}$ NMR (DMSO-d 6 ) $\delta \mathbf{0 . 8 7}(\mathrm{t}$, $\left.3 \mathrm{H}, \mathrm{J}=2.25 \mathrm{~Hz}, \mathrm{CH}_{3}\right), \mathbf{1 . 2 3}\left(\mathrm{m}, 2 \mathrm{H}, \mathrm{CH}_{2}\right), 4.27$ ( t, 2H, J = 7.06 Hz, $\left.\mathrm{CH}_{2}\right), \mathbf{6 . 3 7}$ (bs, 1H, 8-H), 7.48 (d, 1H, J $\left.{ }_{1,2}=2.86 \mathrm{~Hz}, 1-\mathrm{H}\right), 7.56$ (m, 5H, 5-H, 2-H, 3'-H, 4'-H, 5'-H), 7.86 (m, 3H, 4-H, 2' H-, 6'$\mathrm{H}), \mathbf{1 1 , 5 0}(\mathrm{bs}, 1 \mathrm{H}, \mathrm{NH}) ;{ }^{13} \mathrm{C} \mathrm{NMR}\left(\mathrm{DMSO}_{-} \mathrm{d}_{6}\right) \delta 13.89\left(\mathrm{CH}_{3}\right), \mathbf{1 9 . 8 1}\left(\mathrm{CH}_{2}\right), 32.78\left(\mathrm{CH}_{2}\right), 45.80(\mathrm{~N}-$ $\left.\mathrm{CH}_{2}\right), \mathbf{1 9 3 . 5 7}(\mathrm{C}=\mathrm{O}) ; \mathrm{HR}$ MS m/z $317.1720\left[\mathrm{MH}^{+}\right]$.

3-Penthyl-7-phenyl-3H-pyrrolo[3,2-f]quinolin-9-one (29). Yield 86\%; light brown solid; mp 233$235^{\circ} \mathrm{C}$; rf: 0.81 (eluent ethyl acetate/methanol 8:2); ${ }^{1} \mathrm{H}$ NMR $\left(\mathrm{DMSO}_{-} \mathrm{d}_{6}\right) \delta \mathbf{0 . 8 2}(\mathrm{t}, 3 \mathrm{H}, \mathrm{J}=6.86 \mathrm{~Hz}$, $\left.\mathrm{CH}_{3}\right), \mathbf{1 . 2 3}\left(\mathrm{m}, 4 \mathrm{H}, 2 \mathrm{xCH} \mathrm{CH}_{2}\right), \mathbf{1 . 7 8}\left(\mathrm{m}, 2 \mathrm{H}, \mathrm{CH}_{2}\right), 4.28\left(\mathrm{t}, 2 \mathrm{H}, \mathrm{J}=7.06 \mathrm{~Hz}, \mathrm{CH}_{2}\right), 6.37(\mathrm{bs}, 1 \mathrm{H}, 8-\mathrm{H})$, $7.48\left(\mathrm{~d}, 1 \mathrm{H}, \mathrm{J}_{1,2}=2.86 \mathrm{~Hz}, 1-\mathrm{H}\right), 7.56\left(\mathrm{~m}, 5 \mathrm{H}, 5-\mathrm{H}, 2-\mathrm{H} 3^{\prime}-\mathrm{H}, 4^{\prime}-\mathrm{H}, 5^{\prime}-\mathrm{H}\right), 7.86$ (m, 3H, 4-H, 2'-H, 6'-H), 11.50 (bs, 1H, NH); ${ }^{13} \mathrm{C}$ NMR (DMSO-d 6$): \delta 14.25\left(\mathrm{CH}_{3}\right), 22.11\left(\mathrm{CH}_{2}\right), 28.76\left(\mathrm{CH}_{2}\right), 30.37$ $\left(\mathrm{CH}_{2}\right), \mathbf{4 6 . 0 3}\left(\mathrm{N}-\mathrm{CH}_{2}\right), \mathbf{1 8 6 . 3 1}(\mathrm{C}=\mathrm{O}) ; \mathrm{HR} \mathrm{MS} \mathrm{m} / \mathrm{z} 331.1741\left[\mathrm{MH}^{+}\right]$.

3-Cyclopropylmethyl-7-phenyl-3H-pyrrolo[3,2-f]quinolin-9-one (30). Yield 82\%; golden dust; mp 264-266 ${ }^{\circ}$; rf 0.67 (eluent ethyl acetate/methanol 8:2); ${ }^{1} \mathrm{H}$ NMR (DMSO-d 6 ) $\delta \mathbf{0 . 4 1}(\mathrm{m}, 2 \mathrm{H}$, $\left.\mathrm{CH}_{2}\right), \mathbf{0 . 5 3}\left(\mathrm{m}, 2 \mathrm{H}, \mathrm{CH}_{2}\right), \mathbf{1 . 2 6}(\mathrm{m}, 1 \mathrm{H}, \mathrm{CH}), 4.15$ (d, 2H, J = 7.06 Hz, $\left.\mathrm{CH}_{2}\right), 6.37$ (bs, 1H, 8-H), 7.56 (m, 6H, 1-H, 2-H, 5-H, 3'-H, 4'-H, 5'-H), 7.86 (m, 3H, 4-H, 2'-H, 6'-H), 11.50 (bs, 1H, NH); ${ }^{13} \mathrm{C}$ NMR (DMSO- $\left.\mathrm{d}_{6}\right) \delta 4.10\left(\mathrm{CH}_{2}\right), \mathbf{1 2 . 2 2}(\mathrm{CH}), \mathbf{5 0 . 2 2}\left(\mathrm{CH}_{2}\right), \mathbf{1 8 1 . 5 1}(\mathrm{C}=\mathrm{O}) ; \mathrm{HR} \mathrm{MS} \mathrm{m} / \mathrm{z}$ $315.1500\left[\mathrm{MH}^{+}\right]$.

3-Benzyl-7-phenyl-3H-pyrrolo[3,2-f]quinolin-9-one (31). Yield 77\%; golden cristalline solid; mp $330^{\circ} \mathrm{C}$ dec.; Rf: 0.80 (eluent ethyl acetate/methanol 8:2); ${ }^{1} \mathrm{H}$ NMR (DMSO-d $\left.{ }_{6}\right) \delta \mathbf{5 . 7 0}\left(\mathrm{s}, 2 \mathrm{H}, \mathrm{CH}_{2}\right)$, 
$\mathbf{6 . 4 5}$ (bs, 1H, 8-H), 7.40 (m, 5H, 2"-H, 3'”-H, 4"-H, 5'--H, 6"-H), 7.86 (m, 6H, 1-H, 2-H, 4-H, H, 3'-H, 4'-H, 5'-H,), 8.00 (m, 3 H, 5-H, 2'-H, 6'-H), 11.50 (bs, 1H, NH); ${ }^{13} \mathrm{C}$ NMR (DMSO- d 6 ) $\delta$ 49.10 $\left(\mathrm{CH}_{2}\right)$, 193.01 $(\mathrm{C}=\mathrm{O})$. HR MS m/z 351.1579 $\left[\mathrm{MH}^{+}\right]$.

3-Methoxymethyl-7-phenyl-3H-pyrrolo[3,2-f]quinolin-9-one (32). Yield 85\%; grey solid; mp 236-240 ${ }^{\circ}$; rf: 0.69 (eluent ethyl acetate/metanolo 8:2); ${ }^{1} \mathrm{H}$ NMR (DMSO-d $\left.{ }_{6}\right) \delta 3.16\left(s, 3 \mathrm{H}, \mathrm{CH}_{3}\right)$, $\mathbf{5 . 6 3}\left(\mathrm{s}, 2 \mathrm{H}, \mathrm{CH}_{2}\right), \mathbf{6 . 3 7}$ (bs, 1H, 8-H), 7.60 (m, 6H, 1-H, 5-H, 2-H, 3'-H, 4'-H, 5'-H), 7.86 (m, 3H, 4-H, 2'-H, 6'-H), 11.50 (bs, 1H, NH); ${ }^{13} \mathrm{C}$ NMR (DMSO- d 6 ) $\delta \mathbf{5 7 . 2 7}\left(\mathrm{CH}_{3}\right), \mathbf{7 7 . 0 4}$ $\left(\mathrm{CH}_{2}\right), \mathbf{1 8 9 . 1 9}(\mathrm{C}=\mathrm{O}) ; \mathrm{HR} \mathrm{MS} \mathrm{m} / \mathrm{z} 305.1274\left[\mathrm{MH}^{+}\right]$.

\section{Biology}

\section{Cell lines and Culture Conditions}

Human cell lines - thyroid carcinoma (Aro), colon carcinoma (HT-29), pancreas adenocarcinoma (PT-45), hepatocellular carcinoma (Hep G2), ovary carcinoma (Ovcar-3), breast cancer (MCF-7), adrenocortical carcinoma (NCI-H295R), lung carcinoma (A549), uteri cervix carcinoma (Hela) and esophageal squamous carcinoma (OE19 and OE33) - were grown at $37{ }^{\circ} \mathrm{C}$ in a humidified incubator with 5\% $\mathrm{CO}_{2}$. PT-45 and Ovcar-3 cell lines were cultured in RMPI medium supplemented with $10 \%$ heat-inactivated fetal bovine serum (FBS, Invitrogen), $100 \mathrm{U} / \mathrm{mL}$ penicillin $\mathrm{G}$ and $10 \mu \mathrm{g} / \mathrm{mL}$ streptomycin; NCI-H295R were grown in RPMI supplemented with 2\% FBS, 100 $\mathrm{U} / \mathrm{mL}$ penicillin $\mathrm{G}$ and $10 \mu \mathrm{g} / \mathrm{mL}$ streptomycin, $1 \%$ Insulin, Transferrin, Selenium-A (Gibco); Aro, HT-29, Hep G2, MCF-7, A549, Hela, OE19 and OE33 were grown in Dulbecco's modified Eagle's medium supplemented with $10 \%$ heat-inactivated FBS, $100 \mathrm{U} / \mathrm{mL}$ penicillin $\mathrm{G}$ and $10 \mu \mathrm{g} / \mathrm{mL}$ streptomycin. All cell lines were purchased from the American Type Culture Collection. 
FACS histograms. Effects of 7-PPyQs 25-32 on cell cycle progression of A549 cells at concentrations of 1 and $0.1 \mu \mathrm{M}$ concentrations.
Comp.
$1 \mu \mathrm{M}$
$0.1 \mu \mathrm{M}$

25

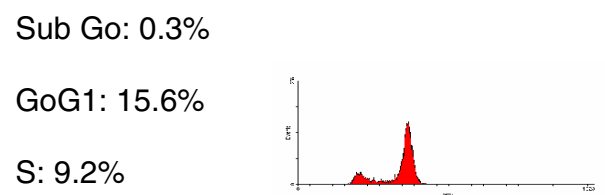

G2/M: $74.9 \%$

Sub Go: $0.4 \%$

26
GoG1: $14.1 \%$

S: $8.1 \%$

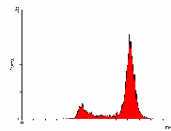

G2/M: $77.3 \%$

Sub Go: $0.3 \%$

27

28

29

30
GoG1: $13.5 \%$

S: $9.6 \%$

G2/M: $76.9 \%$

Sub Go: $0.4 \%$

GoG1: 12.9\%

S: $9.2 \%$

G2/M: $77.6 \%$

Sub Go: $0.4 \%$
GoG1: $15.5 \%$

S: $8.8 \%$

G2/M: $75.5 \%$

Sub Go: $0.3 \%$
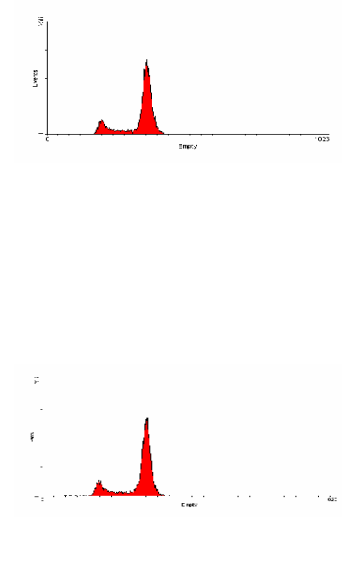

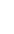

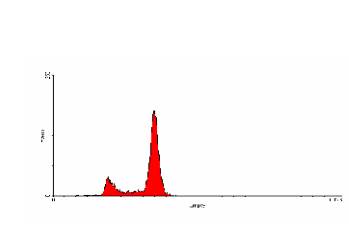

GoG1: $16.3 \%$

S: $8.6 \%$

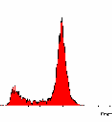

G2/M: $74.6 \%$
Sub Go: $6.4 \%$

GoG1: $66.9 \%$

S: $8.8 \%$

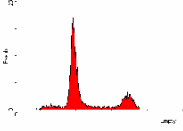

G2/M: $18.2 \%$

Sub Go: $0.6 \%$

GoG1: $11.7 \%$

S: $8.1 \%$

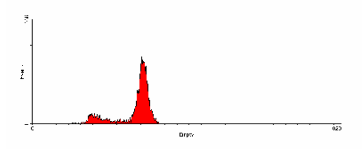

G2/M: $79.8 \%$

Sub Go: $1.3 \%$

GoG1: $13.6 \%$

S: $8.7 \%$

G2/M: $76.2 \%$

Sub Go: $0.2 \%$

GoG1: $11.8 \%$

s: $7.7 \%$

G2/M: $80.4 \%$

Sub Go: $1.0 \%$

GoG1: $12.4 \%$

S: $7.2 \%$

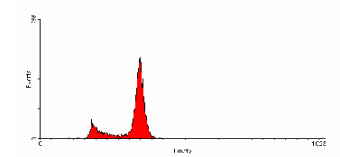

G2/M: 79.6\%

Sub Go: $0.4 \%$

GoG1: $16.5 \%$

S: $7.0 \%$

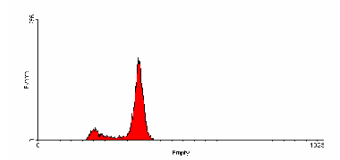

G2/M: $75.7 \%$ 
Sub Go: $0.5 \%$

31

GoG1: $14.6 \%$

S: $9.4 \%$

G2/M: $75.8 \%$

Sub Go: $0.4 \%$

32

GoG1: $13.6 \%$

S: $8.4 \%$

G2/M: $77.9 \%$
Sub Go: $1.1 \%$

GoG1: $78.9 \%$

S: $9.7 \%$

G2/M: $10.4 \%$

Sub Go: $0.5 \%$

GoG1: $81.7 \%$

S: $7.8 \%$

G2/M: $10.2 \%$

Sub Go: $1.2 \%$

Control GoG1: $74.0 \%$

S: $11.8 \%$

G2/M: $13.3 \%$

Sub Go: $0.5 \%$ vincristine

GoG1: $46.8 \%$

S: $7.9 \%$

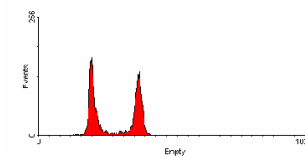

nd

nd

$\mathrm{G}_{2} / \mathrm{M}: 45.2 \%$

\begin{tabular}{|c|c|c|c|c|c|c|c|}
\hline \multicolumn{6}{|c|}{ ELEMENTAL ANALYSIS OF COMPOUNDS 25-32 } \\
\hline \multirow{2}{*}{ Comp } & \multirow{2}{*}{ Formula } & \multicolumn{3}{|c|}{ Calculated (\%) } & \multicolumn{3}{c|}{ Obtained (\%) } \\
\cline { 3 - 8 } & & $\mathrm{C}$ & $\mathrm{H}$ & $\mathrm{N}$ & $\mathrm{C}$ & $\mathrm{H}$ & $\mathrm{N}$ \\
\hline $\mathbf{2 5}$ & $\mathrm{C}_{18} \mathrm{H}_{14} \mathrm{~N}_{2} \mathrm{O}$ & 78.80 & 5.14 & 10.21 & 78.62 & 5.31 & 9.98 \\
\hline $\mathbf{2 6}$ & $\mathrm{C}_{19} \mathrm{H}_{16} \mathrm{~N}_{2} \mathrm{O}$ & 79.14 & 5.59 & 9.72 & 79.47 & 5.85 & 9.45 \\
\hline $\mathbf{2 7}$ & $\mathrm{C}_{20} \mathrm{H}_{18} \mathrm{~N}_{2} \mathrm{O}$ & 79.44 & 6.00 & 9.27 & 79.34 & 6.07 & 9.37 \\
\hline $\mathbf{2 8}$ & $\mathrm{C}_{21} \mathrm{H}_{20} \mathrm{~N}_{2} \mathrm{O}$ & 79.72 & 6.37 & 8.85 & 79.51 & 6.58 & 8.62 \\
\hline $\mathbf{2 9}$ & $\mathrm{C}_{22} \mathrm{H}_{22} \mathrm{~N}_{2} \mathrm{O}$ & 79.97 & 6.71 & 8.48 & 80.20 & 6.74 & 8.64 \\
\hline
\end{tabular}




\begin{tabular}{|c|c|c|c|c|c|c|c|}
\hline 30 & $\mathrm{C}_{21} \mathrm{H}_{18} \mathrm{~N}_{2} \mathrm{O}$ & 80.23 & 5.77 & 8.91 & 80.22 & 5.96 & 8.88 \\
\hline 31 & $\mathrm{C}_{24} \mathrm{H}_{18} \mathrm{~N}_{2} \mathrm{O}$ & 82.26 & 5.18 & 7.99 & 82.18 & 5.17 & 7.72 \\
\hline 32 & $\mathrm{C}_{19} \mathrm{H}_{16} \mathrm{~N}_{2} \mathrm{O}_{2}$ & 74.98 & 5.30 & 9.20 & 74.91 & 5.52 & 8.95 \\
\hline
\end{tabular}

